\title{
Rapid Discrimination of Archaeal Tetraether Lipid Cores by Liquid Chromatography- Tandem Mass Spectrometry
}

\author{
Christopher S. Knappy, James P. J. Chong, ${ }^{\text {b }}$ and Brendan J. Keely ${ }^{\text {a }}$ \\ ${ }^{a}$ Department of Chemistry, University of York, York, United Kingdom \\ ${ }^{\mathrm{b}}$ Department of Biology, University of York, York, United Kingdom
}

\begin{abstract}
Atmospheric pressure chemical ionization liquid chromatography-tandem mass spectrometry (APCI LC-MS/MS) of tetraether lipid cores of archaeal origin reveals distinct dissociation pathways for three classes of core lipid extracted from Methanobacter thermautotrophicus. Within these classes, two isobaric tetraether lipids, one a scarcely reported lipid constituent of $M$. thermautotrophicus and the other an artefact formed during extraction from cultured cells, were identified and distinguished via their MS $^{2}$ spectra. APCI LC-MS/MS discriminates different tetraether core lipid types and isobaric species and reveals the mass of the constituent biphytanyl chains within the tetraether cores, albeit without full elucidation of their structures. Furthermore, the method allows direct estimation of the relative proportions of tetraether core lipids from chromatographic peak area measurement, allowing rapid profiling of these compounds in microbiological and environmental extracts. (J Am Soc Mass Spectrom 2009, 20, 51-59) (C 2009 American Society for Mass Spectrometry
\end{abstract}

A rchaea represent one of the three domains of life, phylogenetically distinct from the eukaryota and eubacteria [1]. They were originally thought to comprise exclusively extremophiles, with each of the three phyla of archaea known at the time (euryarchaeota, crenarchaeota, and korarchaeota) found to thrive in distinct ecological niches such as hot springs, mud volcanoes, and hypersaline lakes. Members of a fourth distinct phylum, the nanoarchaea, have similarly been identified in extreme habitats [2]. By contrast, mesophilic representatives of the crenarchaeota have recently been discovered and shown to be major contributors to the pelagic biomass (up to 20\%) in the world's oceans [3]. Thus far, all identified members of the archaea have been found to contain glycerol diphytanyl diether and/or glycerol dibiphytanyl glycerol tetraether (GDGT) lipids in their cellular membranes [4]. The membrane lipids occur either as naked core lipids, or with phospho- and/or glyco-head groups that are introduced during biosynthesis [5]. The two saturated $\mathrm{C}_{40}$-biphytanyl hydrocarbon chains of the GDGT lipid cores are tethered at both ends to glycerol moieties via ether linkages, with $s n-2,3$ stereochemistry exhibited by each glycerol [6]. The unique structural features of GDGT lipids allow them to be used as specific markers for the archaea, distinguishing this group from eukaryotic and eubacterial lipids, in which sn-1,2 glycerol stereochemistry and ester linkages to unsaturated hy-

Address reprint requests to Dr. B. J. Keely, Department of Chemistry, University of York, Heslington, York YO10 5DD, UK. E-mail: bjk1@ york.ac.uk drocarbon chains are typical. As an example, caldarchaeol (Ia, Figure 1) is structurally the simplest GDGT core lipid and is ubiquitous throughout the archaeal domain. A regioisomer of caldarchaeol, isocaldarchaeol (Ib, Figure 1), has also been identified in three species of archaea, occurring in near equimolar abundance to Ia [7].

Further structural variation among GDGT lipids occurs via incorporation of biphytanyl chains containing between 0 and 4 cyclopentane rings (e.g., $\mathbf{A}+\mathbf{B}$, Figure 2). Despite the potentially large number of biphytanyl structures and combinations possible, only 9 GDGT structures of this type have been identified to date [4]. The expression and abundance of these lipids varies from species to species; the tetraether profiles of methanogenic archaea are often of low diversity and dominated by GDGT I, whereas thermophilic and hyperthermophilic archaea often exhibit a much greater array of GDGT lipids [4, 8]. A novel pair of cyclopentyl ring-containing lipids, crenarchaeol and its regioisomer, have also been identified in cultures of crenarchaeota and in lake and marine sediments $(\mathbf{C}+\mathbf{D}$, Figure 2) [9]. These both contain an additional cyclohexyl ring and are believed to be biomarker compounds specific to the phylum crenarchaeota.

A structural relative of Ia, often referred to as acyclic caldarchaeol (II, Figure 1), is also abundant in the cellular membranes of a significant proportion of the archaea [4]. This core lipid shares the same stereochemistry as Ia in both glycerol units and contains the same $\mathrm{C}_{40}$-biphytanyl linkage between the glycerol units. The second biphytanyl is replaced by two $\mathrm{C}_{20}$-phytanyl 


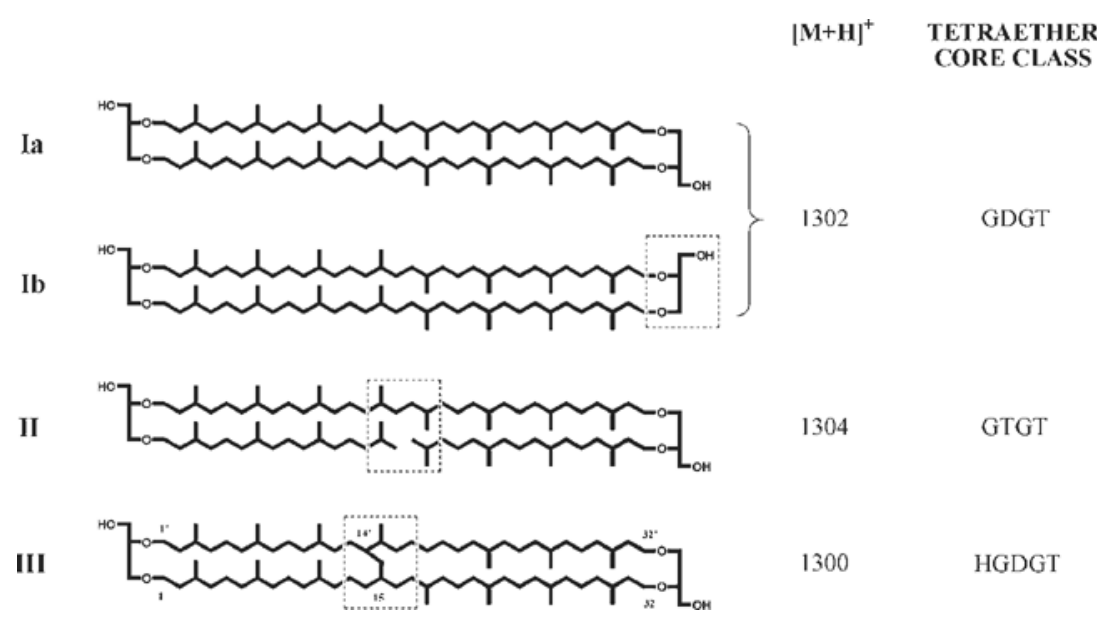
Figure 1. The three classes of tetraether core found in archaea to date, with the nominal $\mathrm{m} / \mathrm{z}$ value of
the protonated molecule indicated in each case; Ia = caldarchaeol, $\mathbf{I b}=$ isocaldarchaeol, $\mathbf{I I}=$ acyclic
caldarchaeol, III = H-shaped caldarchaeol (structure from [13]). Dashed boxes enclose regions that vary from Structure Ia.

chains, giving rise to a glycerol trialkyl glycerol tetraether (GTGT) structure. GTGT II is the only GTGT lipid to have been fully characterized from cultures of archaea profiled to date. Notably, however, a GTGT lipid suggested to contain one cyclopentane ring within the $\mathrm{C}_{40}$-biphytanyl chain has been observed in sediment from a mud volcano [10]. In addition, a series of four GTGT lipids, potentially containing cyclopentane rings within their structures, have been identified in a thermophilic crenarchaeote grown under suboptimal conditions [11]. Although full elucidation of the structures of these lipids is needed, their presence in cultures and environmental samples suggests that GTGT diversity may extend beyond II and match that observed for GDGT lipids.

A third type of core tetraether lipid that is structurally similar to GDGT Ia has been identified in several species of thermophilic and hyperthermophilic methanogens [12]. It consists of a $\mathrm{C}_{80}$-dibiphytanyl hydrocarbon (formed via a covalent linkage between the two $\mathrm{C}_{40}$-biphytanyl chains observed in Ia) capped at both ends with $s n-2,3$ glycerol moieties. The position and nature of the covalent bond linking the two $\mathrm{C}_{40^{-}}$ biphytanyl units is a matter of debate, with evidence for both a C-14' methylene to C-15 methyl link [13] and a C-15' methyl to C-15 methyl link [14] having been reported. Regardless of the precise position of the covalent tether, this lipid has been referred to as $\mathrm{H}$ shaped caldarchaeol (III, Figure 1) on account of the distinctive shape of the hydrocarbon core and its structural similarity to Ia. A series of H-shaped GDGT (HGDGT) lipids containing 0-4 cyclopentyl rings in positions analogous to those observed in GDGT lipids have been identified in the first cultivated thermoacidophilic euryarchaeote that inhabits deep-sea hydrothermal vents [15]. Structurally related $\mathrm{C}_{80}$-dibiphytanyl tetra-acids containing four to eight cyclopentyl rings within the biphytanyl chains have also been observed in naphthenate deposits in crude oil processing equipment [16]. Most likely, tetra-acids of this type are of archaeal origin, suggesting that HGDGT structural variation within the archaeal domain may mimic that of GDGT lipids.

Classically, tetraether lipids from archaeal cultures were identified using two-dimensional thin-layer chromatography (TLC), via comparison of retention factors with those of standards, and quantified by densiometric analysis of stained TLC plates [17]. This method has the advantage of giving a full profile of an archaeal lipidome, including identification of phosphatidyl- and glycosyl-capped lipids, but is severely limited by the range of standards that are available for the purposes of

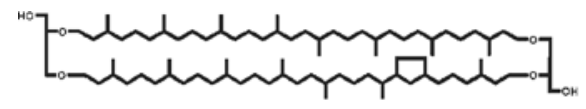

A

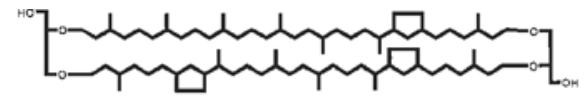

B

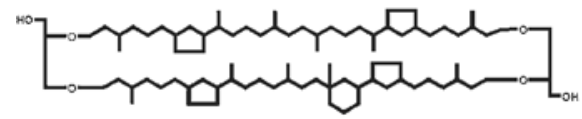

C

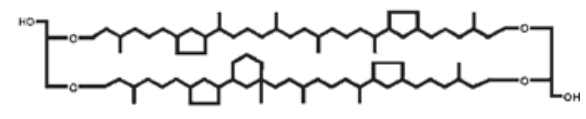

D

Figure 2. Examples of cyclopentyl ring-containing GDGTs. (A) GDGT-1; (B) GDGT-3; (C) crenarchaeol; (D) crenarchaeol regioisomer. 
identification (i.e., new lipids cannot be characterized without subsequent instrumental analysis). In addition, the accuracy of quantification of lipids by densiometric analysis is low by comparison with that possible by use of modern instrumental techniques. As such, methanolytic cleavage of phospho- and glyco-headgroups followed by degradative analysis has often been employed in order for structural characterizations to be made. In particular, treatment of the methanolysis products with $\mathrm{HI}^{-\mathrm{LiAlH}_{4}}$ yields the constituent biphytanyl hydrocarbons of tetraether lipids, which can be identified via gas chromatography-mass spectrometry (GC-MS) [18]. By comparing the mass spectral fragmentation of structures formed in these experiments with those afforded from reduction with deuterated reagents (i.e., $\mathrm{LiAlD}_{4}$ replacing $\mathrm{LiAlH}_{4}$ ), the position of any rings in the chain can be localized and the full structures of the biphytanyl chains determined [4].

Although these methods permit identification of the structures of tetraether lipids with a high degree of certainty, they are cumbersome and require several stages of reaction and separation before instrumental analysis. To circumvent the lengthy analysis times involved, several methods have been developed to analyze GDGT lipids directly from cell culture extracts, the most widely used of these being that developed by Hopmans et al. [10, 19]. The method utilizes atmospheric pressure chemical ionization liquid chromatographymass spectrometry (APCI LC-MS) to analyze GDGT/ GTGT lipids directly from both cellular and sediment extracts. A significant advantage of this method is that the distinct tetraether core lipid components of a particular sample can be separated from one another and analyzed in an intact form (cf. GC-MS where information about the particular combination of biphytanyl chains is lost). Furthermore, the only preparative step required before analysis is chromatographic isolation of the polar lipid fraction on alumina which, coupled with analysis times of less than 30 min for HPLC-MS, leads to significantly greater throughput than can be obtained with other methods. This procedure has been applied extensively to the identification of GDGT core lipids in archaeal culture samples [15], muds [10], and in marine [19], lacustrine [20], and hot-spring sediments [21]. In spite of these advances, studies of the lipids of new archaeal species have, in general, neglected this method in favor of the more established methods mentioned above.

One limitation of the current LC-MS methodology arises from use of only a single stage of mass spectrometric analysis to identify the tetraether lipids. Thus, the lipids are assigned on the basis of their retention times, the mass of the protonated molecule and observable losses of $18\left(-\mathrm{H}_{2} \mathrm{O}\right)$ and $74\left(-\mathrm{C}_{3} \mathrm{H}_{6} \mathrm{O}_{2}\right)$ in the mass spectra. These losses both arise from the glycerol moiety, a common biosynthetic precursor that is not exclusive to tetraether lipids. Furthermore, the neutral fragments lost do not include any of the core hydrocarbon architecture, making it impossible to differentiate be- tween isobaric GDGT lipids that have different constituent biphytanyl partners. Until the biosynthetic pathways for tetraether lipids are fully understood, the potential for the discovery of new GDGT, GTGT, and HGDGT lipids in archaeal species, particularly in novel strains, is significant. Consequently, a rapid method that is able to distinguish between the three core types and to discriminate between isobaric tetraether lipids present in culture samples would prove to be highly beneficial.

Tandem mass spectrometry (MS/MS) has been employed extensively to provide enhanced structural information for $[\mathrm{M}+\mathrm{H}]^{+}$ions formed by "soft" ionization sources such as APCI. By subjecting analytes to collision induced dissociation (CID) and monitoring the product ions formed, specific structural features of the analyte can be determined. Here we discuss the use of liquid chromatography-tandem mass spectrometry (LC-MS/MS) on an ion trap instrument to obtain a greater degree of structural information from tetraether lipids in cultures and environmental samples. Although ion trap mass spectrometry has previously been used to identify the GDGT distributions in sediments from continental Eurasia [22], the use of the tandem MS capabilities of this type of spectrometer has not been reported.

\section{Experimental}

\section{Sample Preparation}

Aerobically harvested frozen cells from a pure culture of Methanobacter thermautotrophicus (previously known as Methanobacterium thermoautotrophicum) [23], grown at $70{ }^{\circ} \mathrm{C}$, were refluxed in methanolic $\mathrm{HCl}(4.8 \mathrm{M}, 4 \mathrm{~h})$ to cleave phosphatidyl and glycosyl headgroups, before liberated core tetraether lipids were partitioned into dichloromethane (DCM). The organic extract was reduced in vacuo and taken to dryness by evaporation under a stream of nitrogen before isolation of tetraether lipids by flash column chromatography on an activated alumina column (approximately $7 \times 50 \mathrm{~mm}$ ), drypacked in a Pasteur pipette and fully solvated with hexane/DCM (9:1 vol/vol). The tetraether extract was dissolved in a minimal amount of DCM, loaded onto the column and washed with three bed volumes of hexane/DCM (9:1 vol/vol) to remove apolar components. Tetraether species were eluted with three bed volumes of DCM/methanol (1:1 vol/vol). The solvent was removed in vacuo and the sample was dissolved by sonication for $5 \mathrm{~min}$ in hexane/propan-2-ol (99:1 vol/ vol) before instrumental analysis.

\section{Instrumentation}

The purified tetraether extract was analyzed using a Finnigan (Thermo-Finnigan, San Jose, CA) system comprising a Thermo Separations AS3000 autosampler, P4000 gradient pump, and a Finnigan MAT LCQ ion 
trap mass spectrometer equipped with an APCI ionization source. Separation was achieved using a previously described literature method [10], which uses a binary solvent system ( $\mathrm{A}=$ hexane, $\mathrm{B}=$ propan-2-ol) operated

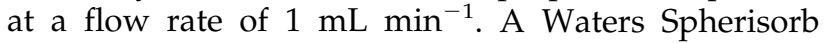
S5- $\mathrm{NH}_{2}$ column $(4.6 \times 250 \mathrm{~mm}, 5 \mu \mathrm{m}$; Elstree, UK) was eluted isocratically for $5 \mathrm{~min}$ with $99 \% \mathrm{~A}, 1 \% \mathrm{~B}$, followed by a linear gradient to $2 \% \mathrm{~B}$ in $56.25 \mathrm{~min}$. The column was backflushed with $90 \% \mathrm{~A}, 10 \% \mathrm{~B}$ for $10 \mathrm{~min}$, and reconditioned to the starting composition for $15 \mathrm{~min}$ before reuse. The injection volume was fixed at $5 \mu \mathrm{L}$.

Eluting species were monitored using the positive ionization mode of the APCI source. The mass spectral response was optimized via tuning on the $[\mathrm{M}+\mathrm{H}]^{+}$ peak for $\mathbf{I}(m / z$ 1302) during direct infusion of a portion of the $M$. thermautotrophicus $[\mathrm{MTH}(\Delta H)]$ extract. I is the dominant lipid in $\operatorname{MTH}(\Delta H)$ and, hence, the extract can be used directly for instrument tuning, avoiding the need for preparation of a pure tetraether standard. The APCI parameters found to be optimal are as follows: vaporizer temperature $450{ }^{\circ} \mathrm{C}$, sheath gas $\left(\mathrm{N}_{2}\right)$ pressure 19 (arb. units), auxiliary gas $\left(\mathrm{N}_{2}\right)$ pressure 30 (arb. units), capillary temperature $150{ }^{\circ} \mathrm{C}$, capillary voltage $47.8 \mathrm{kV}$, corona discharge current $5 \mu \mathrm{A}$, corona discharge voltage $5 \mathrm{kV}$. Positive ion MS spectra were obtained by scanning a narrow mass range from $\mathrm{m} / \mathrm{z}$ 1280 to $1320 . \mathrm{MS}^{2}$ spectra were recorded using the data dependent ion scan feature, in which the base peak of an MS scan is selected for collision induced dissociation (CID) in $\mathrm{MS}^{2}$. The dwell time was set at $100 \mathrm{~ms}$ and the collision energy to $41 \%$. The isolation width was set to $3 \mathrm{~m} / \mathrm{z}$, the minimum value found to be required to enable tetraether lipids to be trapped. $\mathrm{MS}^{3}$ spectra were generated using identical CID conditions following isolation of the base peak ion from $\mathrm{MS}^{2}$.

MS and $\mathrm{MS}^{2}$ spectra are labeled with average $\mathrm{m} / \mathrm{z}$ values as measured on the ion trap over 12 LC-MS/MS analyses. Owing to the large mass defect in tetraether lipids and product ions generated from them during CID, these values can be up to $1.1 \mathrm{~m} / \mathrm{z}$ units larger than the nominal $\mathrm{m} / \mathrm{z}$ value for the ion in question. Previous reports of the LC-MS analysis of tetraether lipids have quoted the measured $\mathrm{m} / \mathrm{z}$ values of the protonated molecules to the nearest integral value [10, 19]. That approach can, however, lead to ambiguities when applied to product ions observed in the $\mathrm{MS}^{2}$ spectra of the lipids, particularly in cases where the product ion has an $m / z$ value that is median to two integral values. Hence, discussion of tetraether dissociation during CID is restricted to the nominal $\mathrm{m} / \mathrm{z}$ values of the product ions observed and the nominal masses of neutral molecules lost during their formation.

\section{Results and Discussion}

\section{LC-MS}

The MS base peak chromatogram of the extract of $\operatorname{MTH}(\Delta H)$ contains four peaks, with retention times $\left(t_{\mathrm{R}}\right)$ of $7,16,23$, and $35 \mathrm{~min}$ and having base peak ions at $\mathrm{m} / \mathrm{z}$ 1316.0, 1303.9, 1302.0, and 1299.9, respectively. Reconstructed mass chromatograms for the four distinct base peak ions (Figure 3a) revealed an additional species with $[\mathrm{M}+\mathrm{H}]^{+}$at $m / z 1316.0\left(t_{\mathrm{R}}=22 \mathrm{~min}\right)$ that had been concealed by partial coelution with a species with $[\mathrm{M}+$ $\mathrm{H}^{+}$at $m / z 1302.0$ in the MS base peak chromatogram. a)

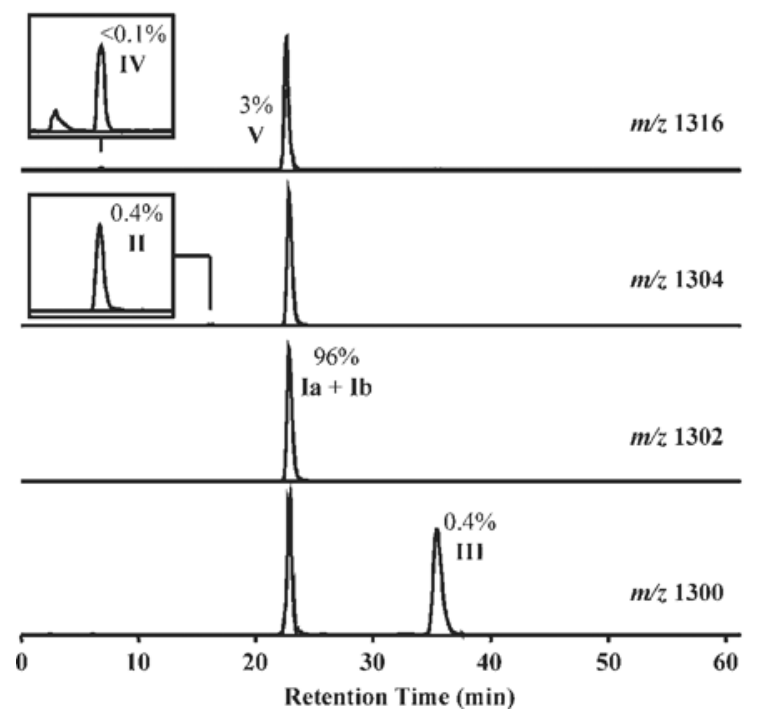

b)

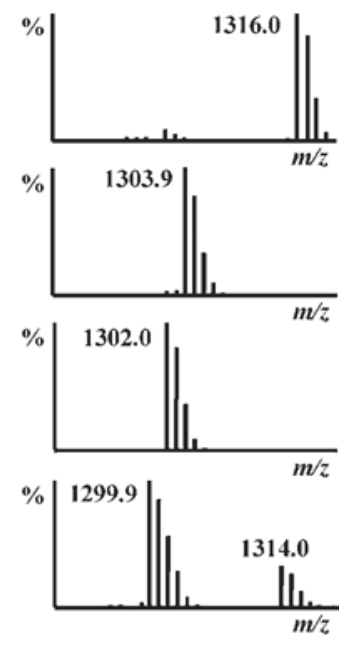

Figure 3. (a) Reconstructed mass chromatograms for the four base peak ions in the positive ion MS base peak chromatogram. Each chromatogram was generated by selecting all ions within $\mathrm{m} / \mathrm{z} \pm 0.5$ of the base peak in question from the total MS dataset. Insets show magnifications of small peaks. The percentage of the total extract contributed by each lipid is also shown. The peak at $23 \mathrm{~min}$ in the $\mathrm{m} / \mathrm{z}$ 1304 mass chromatogram corresponds to an isotopic peak of GDGTs Ia and Ib. (b) Representative mass spectra for lipids I-III and V. Lipid IV gave a mass spectrum identical to that of $\mathbf{V}$. 
The latter corresponds to the protonated molecule of GDGT Ia, which has been observed previously by LC-MS with a similar retention time. Likewise, an early eluting peak with $[\mathrm{M}+\mathrm{H}]^{+}$at $m / z$ 1303.9, corresponding to the protonated molecule of GTGT II, has been observed previously under similar conditions [10]. LC-MS detection of $\mathbf{I b}$ has not been reported, but the peak in the $m / z 1302$ mass chromatogram shows the greatest asymmetry of all of the peaks identified (asymmetry factor $=2.9$, calculated as the ratio of the peak median to peak tail and peak median to peak front distances) suggesting that Ib coelutes with Ia under the chromatographic conditions employed. The component with a protonated molecule at $m / z 1299.9$ exhibits a very different retention time to that of an isobaric GDGT containing one cyclopentane ring in its core structure (Structure A in Figure 2, observed to elute $\sim 1$ min later than Ia in many LC-MS studies, including analyses performed in our laboratory) [10]. HGDGT III has previously been identified as a minor component in a TLC analysis of the tetraether lipids in this archaeon [13], and was observed to elute late in an LC-MS analysis of the tetraether lipids of a thermoacidophile [15]. Accordingly, the latest eluting component in the base peak chromatogram is assigned as HGDGT III. The two lipids observed to have protonated molecules at $m / z 1316.0\left(\mathbf{I V}, t_{\mathrm{R}}=7 \mathrm{~min}\right.$ and $\left.\mathbf{V}, t_{\mathrm{R}}=22 \mathrm{~min}\right)$ have not, to the best of our knowledge, been identified previously via LC-MS analysis.

Representative mass spectra for tetraether lipids I-V (Figure $3 b$ ) show that these species all exhibit base peak ions corresponding to the protonated molecules ([M + $\mathrm{H}]^{+}$) in MS analysis, a feature that has been observed previously [10]. Consequently, it was possible to determine the relative abundances of the five tetraether lipids in the extract by integration of peak areas in the relevant reconstructed mass chromatogram. The lipid extract is dominated by caldarchaeol isomers Ia and I Ib, which contributed $96 \%$ of the total tetraether component. Lipid V contributes 3\% of the total extract and lipids II-IV contribute only minor amounts to the total lipid mass ( $<1 \%$ combined). The mass spectrum obtained over the elution time of lipid III highlights the presence of a coeluting species that has a protonated molecule at $m / z$ 1314.0. The isotopic profile of this species matches that observed for lipids I-V, suggesting a tetraether structure. As an $\mathrm{MS}^{2}$ spectrum could not be generated for this compound under the operational scan range chosen the likely structure of this species is not discussed.

\section{Tandem MS Analysis}

Due to the close structural relation of lipids I-III, the product ions generated in the ion trap from the CID of the protonated molecules show distinct similarities, albeit reflecting the $2 \mathrm{Da}$ differences in the precursor protonated molecules. Most notable is the presence of three distinct spectral regions in the $\mathrm{MS}^{2}$ spectra of lipids I-III (Figure 4). Region $1(\mathrm{~m} / \mathrm{z}>1150)$ results from loss of small neutral molecules (water and propenal), Region 2 (m/z 900-1025) from loss of one phytanyl group with accompanying loss of small neutral molecules, and Region $3(\mathrm{~m} / \mathrm{z} 550-850)$ from loss of either one biphytanyl group or two phytanyl groups together with small neutral molecules. The ranges for the three regions have been selected to encompass all product ions likely to be generated from the known tetraether lipids identified to date. The number of these regions in which product ions are observed in the $\mathrm{MS}^{2}$ spectrum of the tetraether lipid analyzed is indicative of the specific structural core type exhibited by the lipid. The CID spectra of lipids I-III are discussed individually and summarized (Table 1).

\section{$M S^{2}$ of GDGT I}

On account of the coelution of the isobaric GDGTs Ia and $\mathbf{I b}$, both are indiscriminately trapped and subjected to CID, unavoidably producing overlap of the product ions from both species in the resultant $\mathrm{MS}^{2}$ spectrum (Figure 4a). In this initial discussion of the dissociation of protonated caldarchaeol, it is assumed that $\mathbf{I a}$ and $\mathbf{~ I b}$ undergo identical dissociations and the two species are discussed together as GDGT I in the interests of clarity. The $\mathrm{MS}^{2}$ spectrum of I shows two distinct groups of product ions, in Regions 1 and 3, respectively. The former includes a number of product ions resulting from small neutral losses from one of the terminal glycerol moieties in I. As has been reported previously for GDGT I [10], product ions at $m / z 1283\left(-18 ; \mathrm{H}_{2} \mathrm{O}\right)$ and $m / z 1227\left(-74 ; \mathrm{C}_{3} \mathrm{H}_{6} \mathrm{O}_{2}\right)$ result from loss from/of this terminal functionality.

A number of product ions were observed to arise from additional losses of one or two molecules of water from both $\mathrm{m} / \mathrm{z} 1283$ and 1227. Interestingly, a product ion at $m / z 1245$ was also observed and is tentatively assigned as being formed via loss of propenal $\left(\mathrm{C}_{3} \mathrm{H}_{4} \mathrm{O}\right)$ from one end of the protonated caldarchaeol molecule. The existence of this product ion suggests that the ion at $m / z 1227$ in the $\mathrm{MS}^{2}$ spectrum of I may be formed in two stages, with separate losses of water and propenal, as opposed to being formed in one stage via the loss of a $\mathrm{C}_{3} \mathrm{H}_{6} \mathrm{O}_{2}$ unit. Support for this assertion comes from the presence of a product ion at $m / z 1227$ in the $\mathrm{MS}^{3}$ spectrum of I following isolation and CID of the $\mathrm{m} / \mathrm{z}$ 1283 species formed in $\mathrm{MS}^{2}$. It is important to note that although $\mathrm{MS}^{3}$ analysis proves useful in this case, we found in general that the $\mathrm{MS}^{3}$ spectra were of significantly lower signal strength than the $\mathrm{MS}^{2}$ spectra. Furthermore, most of the $\mathrm{MS}^{3}$ spectra did not contain product ions that were not also observed in $\mathrm{MS}^{2}$ and consequently, routine $\mathrm{MS}^{3}$ analysis proved redundant for characterization of tetraether cores.

Region 3 of the $\mathrm{MS}^{2}$ spectrum shows product ions arising from loss of one of the two biphytanyl hydrocarbon chains in $\mathbf{I}$. The product ion at $m / z 743$ is formed 

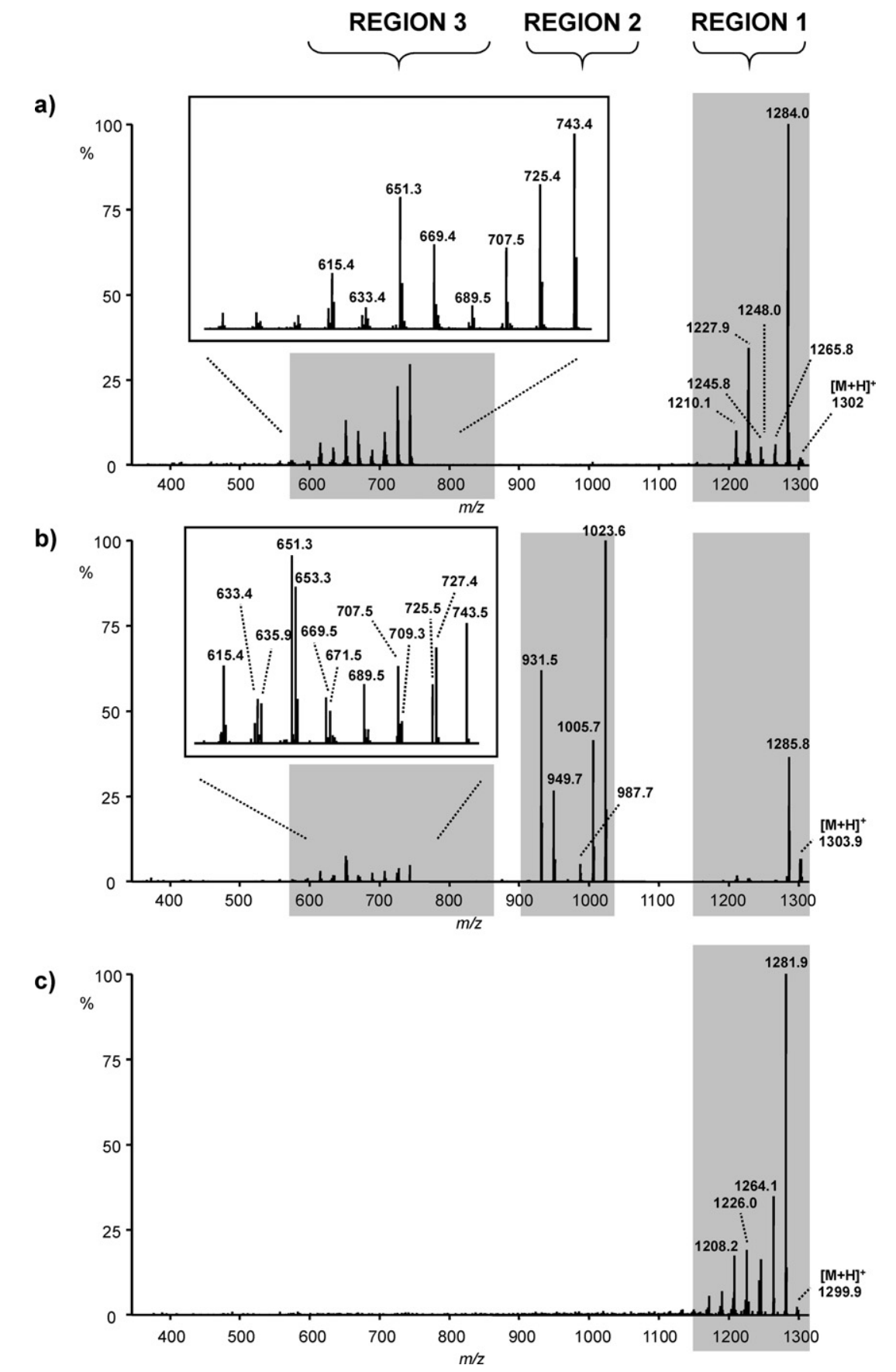

Figure 4. $\mathrm{MS}^{2}$ spectra for lipids I-III of $\mathrm{MTH}(\Delta H)$. Insets show expansions of Region 3 of the spectra

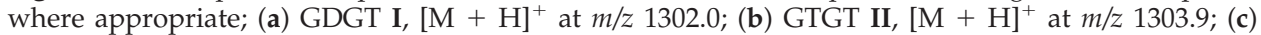
HGDGT III, $[\mathrm{M}+\mathrm{H}]^{+}$at $m / z 1299.9$.

via loss of 1,31-biphytadiene from the protonated molecule of $\mathbf{I}$, with double hydrogen transfer back to the charge-retaining species. This product ion is of particular significance as it allows, for the first time, the precise mass of the alkyl chains of an intact tetraether lipid to be deduced. Further product ions in this region also form as a result of loss of biphytadiene, but show concomitant losses of 1-4 molecules of water and/or loss of propenal.
$M S^{2}$ of GTGT II

The $\mathrm{MS}^{2}$ spectrum of GTGT II (Figure $4 \mathrm{~b}$ ) shows similarities to that of I. In this case, however, product ions are observable in Regions 1, 2, and 3. Several ions in Regions 1 and 2 correspond to fragment ions observed in single-stage mass spectrometry of II [10].

Region 1 of the $\mathrm{MS}^{2}$ spectrum is dominated by a product ion at $m / z 1285$, corresponding to a species in 
Table 1. Product ions generated in each of the three distinct regions of the $\mathrm{MS}^{2}$ spectra following CID of the $[\mathrm{M}+\mathrm{H}]^{+}$ions of tetraether lipids I-III

\begin{tabular}{|c|c|c|c|c|c|}
\hline & & Number of neutral framentc loct & $\begin{array}{r}m / z \\
\mathrm{ge}\end{array}$ & $\begin{array}{l}\text { pondin } \\
\text { from [N }\end{array}$ & $\begin{array}{l}t \text { ion } \\
f^{c}\end{array}$ \\
\hline & Mass loss $(\mathrm{Da})^{\mathrm{a}}$ & {$\left[\mathrm{H}_{2} \mathrm{O}, \mathrm{C}_{3} \mathrm{H}_{4} \mathrm{O}, \mathrm{C}_{20} \mathrm{H}_{40}, \mathrm{C}_{40} \mathrm{H}_{78}\right]^{\mathrm{b}}$} & I & II & III \\
\hline$[\mathrm{M}+\mathrm{H}]^{+}$ & -0 & {$[0,0,0,0]$} & 1301 & 1303 & 1299 \\
\hline Region 1 & -18 & {$[1,0,0,0]$} & 1283 & 1285 & 1281 \\
\hline & -36 & {$[2,0,0,0]$} & 1265 & 1267 & 1263 \\
\hline & -54 & {$[3,0,0,0]$} & 1247 & & 1245 \\
\hline & -56 & {$[0,1,0,0]$} & 1245 & & 1243 \\
\hline & -74 & {$[1,1,0,0]$} & 1227 & & 1225 \\
\hline & -92 & {$[2,1,0,0]$} & 1209 & & 1207 \\
\hline & -110 & {$[3,1,0,0]$} & & & 1189 \\
\hline & -128 & {$[4,1,0,0]$} & & & 1171 \\
\hline Region 2 & -280 & {$[0,0,1,0]$} & & 1023 & \\
\hline & -298 & {$[1,0,1,0]$} & & 1005 & \\
\hline & -316 & {$[2,0,1,0]$} & & 987 & \\
\hline & -354 & {$[1,1,1,0]$} & & 949 & \\
\hline & -372 & {$[2,1,1,0]$} & & 931 & \\
\hline Region 3 & -558 & {$[0,0,0,1]$} & 743 & & \\
\hline & -560 & {$[0,0,2,0]$} & & 743 & \\
\hline & -576 & {$[1,0,0,1]$} & 725 & 727 & \\
\hline & -578 & {$[1,0,2,0]$} & & 725 & \\
\hline & -594 & {$[2,0,0,1]$} & 707 & 709 & \\
\hline & -596 & {$[2,0,2,0]$} & & 707 & \\
\hline & -612 & {$[3,0,0,1]$} & 689 & & \\
\hline & -614 & {$[3,0,2,0]$} & & 689 & \\
\hline & -632 & {$[1,1,0,1]$ or $[4,0,2,0]$} & 669 & 671 & \\
\hline & -634 & {$[1,1,2,0]$} & & 669 & \\
\hline & -650 & {$[2,1,0,1]$} & 651 & 653 & \\
\hline & -652 & {$[2,1,2,0]$} & & 651 & \\
\hline & -668 & {$[3,1,0,1]$} & 633 & 635 & \\
\hline & -670 & {$[3,1,2,0]$} & & 633 & \\
\hline & -686 & {$[4,1,0,1]$} & 615 & & \\
\hline & -688 & {$[4,1,2,0]$} & & 615 & \\
\hline
\end{tabular}

a Mass loss from $[\mathrm{M}+\mathrm{H}]^{+}$. Values quoted are the nominal masses of the neutral molecules lost.

b Number of each of four types of neutral loss occurring; [water, propenal, phytene, 1,31-biphytadiene].

${ }^{c}$ Values quoted are the nominal $\mathrm{m} / \mathrm{z}$ values for the product ion observed.

which a molecule of water has been lost from the protonated molecule of II. Region 2 of the spectrum reveals product ions in which one of the two constituent phytanyl chains of II has been lost during CID. The peak at $m / z 1023$ represents a product ion formed by loss of phytene $\left(\mathrm{C}_{20} \mathrm{H}_{40}\right)$ with proton transfer to the charge-retaining species. Other product ions in this region have also lost phytene, but have suffered additional losses of propenal and/or 1 to 2 molecules of water. Region 3 of the $\mathrm{MS}^{2}$ spectrum shows a series of product ions in which the second phytanyl chain has also been lost from II, again via elimination of $\mathrm{C}_{20} \mathrm{H}_{40}$ with proton transfer back to the charge-retaining species. Loss of this second alkyl group generates product ions that are structurally identical to those produced when a biphytanyl chain is lost during CID of I and, as such, this spectral region exhibits essentially the same ions as are observed in the equivalent region of the $\mathrm{MS}^{2}$ spectrum of I. Notably, however, a second related series of product ions at higher $m / z$ ( +2 units) is also apparent in this region. These ions are consistent with loss of a molecule of biphytadiene (cf. the mechanism of loss of biphytadiene from I), with or without additional loss of propenal or water, though such losses would be expected to show a concomitant loss of a phytanyl group. The precise mechanism for formation of this product ion series is unclear, but could be explained by a rearrangement in which a phytanyl group has been transposed to elsewhere on the architecture before loss of biphytadiene.

\section{$M S^{2}$ of HGDGT III}

The $\mathrm{MS}^{2}$ spectrum of HGDGT III (Figure 4c) reveals product ions that are formed via the same losses that generated the product ions seen in the $\mathrm{MS}^{2}$ spectra of tetraethers I and II. The overall degree of dissociation for III is far less pronounced than for the other tetraethers, with product ions being present only in spectral Region 1 on account of the covalent link between the two hydrocarbon chains in this molecule. Provided that this $\mathrm{C}-\mathrm{C}$ bond remains intact during dissociation, any product ion generated will maintain a $\mathrm{C}_{80}$ hydrocarbon core, resulting in loss of small neutral molecules 
only. This tethering of the biphytanyl chains, however, allows more extensive loss of functionality from both glycerol groups and consequently, product ion peaks relating to loss of propenal and/or one to four molecules of water are observed in Region 1 of the $\mathrm{MS}^{2}$ spectrum of III.

\section{$M S^{2}$ of Tetraether Lipids IV and $\mathbf{V}$}

From the full-MS base peak chromatogram of unknown lipids IV and $\mathbf{V}$ it is evident that they both have protonated molecules with nominal $\mathrm{m} / \mathrm{z} 1315$. The $14 \mathrm{Da}$ increase relative to GDGT I suggests that these lipids might be higher homologues of I (i.e., $\mathrm{C}_{87} \mathrm{H}_{174} \mathrm{O}_{6}$ tetraether lipids), although the nature of this homologation, the position of the additional carbon and the core lipid structural type, can not be ascertained from singlestage mass spectral analysis.

The $\mathrm{MS}^{2}$ spectra generated from CID of the protonated molecules of IV and V (Figure 5) only exhibit product ions in Regions 1 and 3, suggesting that both structures are GDGT lipids. The $\mathrm{MS}^{2}$ spectral Region 3, relating to product ions in which an alkyl chain has been lost, provides significant information about the location of the additional carbon atom in IV and $\mathbf{V}$. GDGTs IV and $\mathbf{V}$ both show a product ion at $m / z 757$ in their $\mathrm{MS}^{2}$ spectra, corresponding to loss of biphytadiene $\left(\mathrm{C}_{40} \mathrm{H}_{78}\right)$ from the protonated molecule. Notably, only GDGT V exhibits a product ion at $m / z 743$ arising from loss of $\mathrm{C}_{41} \mathrm{H}_{80}$, indicating that GDGT $\mathbf{V}$ is a structure based on caldarchaeol in which one of the alkyl chains has an additional carbon. Previous studies exploring the biosynthetic pathways of the tetraethers in $\operatorname{MTH}(\Delta H)$ revealed production of an unexpected
$\mathrm{C}_{87} \mathrm{H}_{174} \mathrm{O}_{6}$ GDGT lipid homologue of $\mathrm{I}$, which was named homocaldarchaeol [24]. Isotopic enrichment ${ }^{13} \mathrm{C}$ NMR studies determined that the additional carbon in this lipid was a methyl group situated on the $\mathrm{C}-13$ position of one of the two biphytanyl chains of I (structure shown in Figure 5). As such, it is likely that GDGT V determined in these studies corresponds to homocaldarchaeol.

The absence of a product ion at $m / z 743$ in the $\mathrm{MS}^{2}$ spectrum of GDGT IV is consistent with a structure in which only $\mathrm{C}_{40} \mathrm{H}_{78}$ alkyl groups are present, indicating that the additional carbon atom in this lipid must be incorporated into one of the two capping glycerol groups. The $\mathrm{MS}^{2}$ spectral Region 1, in which only terminal functionality is lost from the protonated molecules of IV and V, supports both of these assertions. Product ions at $m / z 1297$ and 1279, relating to loss of one or two molecules of water are observed in the $\mathrm{MS}^{2}$ spectrum of $\mathbf{V}$, wholly consistent with losses from the glycerol functionality observed during CID of I. GDGT IV, on the other hand, dissociates to give a product ion at $m / z 1297$ relating to loss of one molecule of water, but does not undergo a subsequent loss of a second molecule of water. It does, however, generate a product ion at $m / z$ 1283, consistent with a loss of methanol. As such, it is highly likely that GDGT IV represents a caldarchaeol molecule methyl etherified at the primary hydroxyl of one of its glycerol head groups. All other product ions observed in the $\mathrm{MS}^{2}$ spectrum of IV are consistent with this assignment. This species is likely to have been generated as an artefact during the acidic methanolysis of the $\operatorname{MTH}(\Delta H)$ culture material. Previous studies of the products of methanolysis of bacteriochlorophylls within our group have shown the methyl etherification of a secondary hydroxyl, suggesting that a)
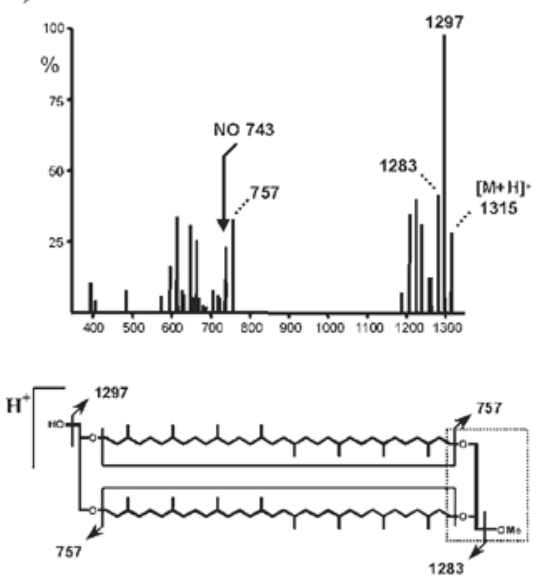

IV b)
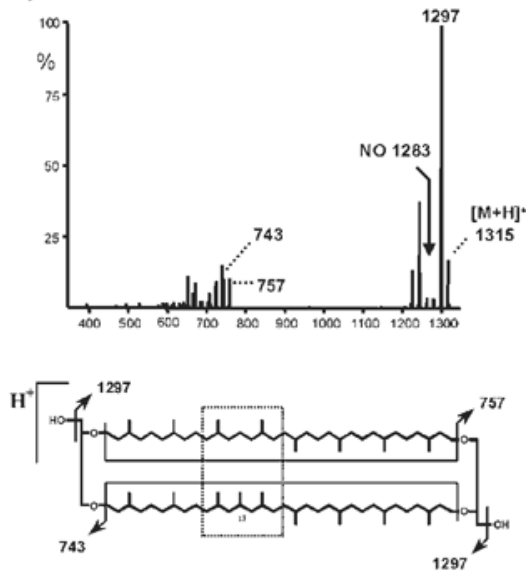

V

Figure 5. $\mathrm{MS}^{2}$ spectra of GDGT lipids having protonated molecules at nominal $\mathrm{m} / \mathrm{z} 1315$, showing the characteristic product ions and dissociations responsible for the generation of these ions. Dashed boxes highlight the position of the additional carbon atom (Ia) in each case; (a) GDGT IV ( $t_{\mathrm{R}}=7 \mathrm{~min}$ ). The absence in this spectrum of a product ion at $m / z 743$ is highlighted; (b) GDGT V $\left(t_{\mathrm{R}}=22 \mathrm{~min}\right)$. The absence in this spectrum of a product ion at $\mathrm{m} / \mathrm{z} 1283$ is highlighted. The structure of $\mathbf{V}$ shown is a tentative structural assignment on the basis of a previous report of a GDGT lipid homologue of I, homocaldarchaeol, with a methyl group replacing a hydrogen at the C-13 position [24]. 
there is precedent for nucleophilic substitution of free hydroxyl groups during acidic methanolysis [25]. To test this assertion, an ethanolytic cleavage was used in place of methanolysis and GDGT IV was no longer observable in the MS base peak chromatogram of the lipid extract. As such, clearly GDGT IV is generated from I during the extraction of $\operatorname{MTH}(\Delta H)$ and does not naturally occur in this archaeon.

\section{Conclusions}

Analysis of the lipid extract of a methanogenic archaeon, $\operatorname{MTH}(\Delta H)$, by APCI LC-MS/MS has revealed the presence of three core lipid structural types; a GTGT lipid, a HGDGT lipid, and three GDGT lipids. CID of the protonated molecules of each of the three tetraether types generated a series of product ions via losses of water, propenal, and alkene groups, providing significant enhancement in characterization potential compared to analysis of tetraether lipids via single-stage MS. The MS ${ }^{2}$ spectra of caldarchaeol (GDGT), acyclic caldarchaeol (GTGT), and H-shaped caldarchaeol (HGDGT) exhibit between one and three distinct spectral regions relating to the number of alkyl groups that can readily be lost. Thus, application of tandem mass spectrometry readily distinguishes between the three core types, and work is underway to verify that these patterns are consistent in other tetraether lipids. Examination of the product ions in the $\mathrm{MS}^{2}$ spectra of the two isobaric GDGT lipids with $[\mathrm{M}+\mathrm{H}]^{+}$at $\mathrm{m} / \mathrm{z} 1315$ provided significant information about their structures. In particular, product ions formed by loss of one alkyl chain reveal that one lipid is a naturally occurring methylated homologue of caldarchaeol, in which the extra carbon atom is located on one of the biphytanyl chains, and the other is a methyl ether of caldarchaeol formed during extraction. These structural assignments highlight the potential of LC-MS/MS for differentiating isobaric intact tetraether core lipids not only using quadrupole ion trap spectrometry, but potentially on other instrumentation with multistage mass spectral capabilities, and suggest that the method may be useful in the identification of novel tetraether lipids discovered in the future.

\section{Acknowledgments}

The authors acknowledge support for this study by a scholarship from the University of York. Two anonymous reviewers are thanked for their helpful and constructive critique.

\section{References}

1. Fox, G. E.; Stackebrandt, E.; Hespell, R. B.; Gibson, J.; Maniloff, J.; Dyer, T. A.; Wolfe, R. S.; Balch, W. E.; Tanner, R. S.; Magrum, L. J.; Zablen, L. B.; Blakemore, R.; Gupta, R.; Bonen, L.; Lewis, B. J.; Stahl, D. A.; Luehrsen, K. R.; Chen, K. N.; Woese, C. R. The Phylogeny of Prokaryotes. Science 1980, 209, 457-463.
2. Huber, H.; Hohn, M. J.; Rachel, R.; Fuchs, T.; Wimmer, V. C.; Stetter K. O. A New Phylum of Archaea Represented by a Nanosized Hyperthermophilic Symbiont. Nature 2002, 417(6884), 63-67.

3. Karner, M. B.; DeLong, E.F.; Karl, D. M. Archaeal Dominance in the Mesopelagic Zone of the Pacific Ocean. Nature 2001, 409, 507-510.

4. De Rosa, M.; Gambacorta, A. The Lipids of Archaebacteria. Prog. Lipid Res. 1988, 27, 153-175.

5. Koga, Y.; Morii, H. Biosynthesis of Ether-Type Polar Lipids in Archaea and Evolutionary Considerations. Microbiol. Mol. Biol. Rev. 2007, 71(1), 97-120.

6. Kates, M. Ether Lipids in Extremely Halophilic Bacteria. In Ether Lipids: Chemistry and Biology, Snyder. F., Ed.; Academic Press: New York, 1972; p. 351.

7. Gräther, O.; Arigoni, D. Detection of Regioisomeric Macrocyclic Tetraethers in the Lipids of Methanobacterium thermoautotrophicum and other archaeal organisms. I. Chem. Soc. Chem. Commun. 1995, 405-406.

8. Koga, Y.; Nishihara, M.; Morii, H.; Akagawa-Matsushita, M. Ether Polar Lipids of Methanogenic Bacteria-Structures, Comparative Aspects, and Biosyntheses. Microbiol. Rev. 1993, 57(1), 164-182.

9. Sinninghe Damsté, J. S.; Schouten, S.; Hopmans, E. C.; van Duin, A. C. T.; Geenevasen, J. A. J. Crenarchaeol: The Characteristic Core Dibiphytanyl Glycerol Tetraether Membrane Lipid of Cosmopolitan Pelagic Crenarchaeota. J. Lipid Res. 2002, 43, 1641-1651.

10. Hopmans, E. C.; Schouten, S.; Pancost, R. D.; van der Meer, M. T. J. Sinninghe Damsté, J. S. Analysis of Intact Tetraether Lipids in Archaea Cell Material and Sediments by High Performance Liquid Chromatography/Atmospheric Pressure Chemical Ionization Mass Spectrometry. Rapid Commun. Mass Spectrom. 2000, 14, 585-589.

11. de la Torre, J. R.; Walker, C. B.; Ingalls, A. E.; Könneke, M.; Stahl, D. A. Cultivation of a Thermophilic Ammonia Oxidizing Archaeon Synthesizing Crenarchaeol. Environ. Microbiol. 2008, 10(1), 810-818.

12. Sugai, A.; Uda, I.; Itoh, Y. H., Itoh, T. The Core Lipid Composition of the 17 Strains of Hyperthermophilic Archaea, Thermococcales. J. Oleo Sci. 2004, 53(1), 41-44.

13. Morii, H.; Eguchi, T.; Nishihara, M.; Kakinuma, K.; Konig, H.; Koga, Y. A Novel Ether Core Lipid with H-Shaped $\mathrm{C}_{80}$-Isoprenoid Hydrocarbon Chain from Hyperthermophilic Methanogen Methanothermus fervidus. Biochim. Biophys. Acta 1998, 1390, 339-345.

14. Lutnaes, B. F.; Krane, J.; Smith, B. E.; Rowland, S. J. Structure Elucidation of $\mathrm{C}_{80}, \mathrm{C}_{81}$, and $\mathrm{C}_{82}$ Isoprenoid Tetra-Acids Responsible for Naphthenate Deposition in Crude Oil Production. Org. Biomol. Chem. 2007, 5(12), 1873-1877.

15. Schouten, S.; Baas, M.; Hopmans, E. C.; Reysenbach, A. L.; Damsté, J. S. S. Tetraether Membrane Lipids of Candidatus Aciduliprofundum boonei, a Cultivated Obligate Thermoacidophilic Euryarchaeote from Deep-Sea Hydrothermal Vents. Extremophiles 2008, 12(1), 119-124.

16. Lutnaes, B. F.; Brandal, Ø.; Sjöbolm, J.; Krane, J. Archaeal $C_{80}$ Isoprenoid Tetra-Acids Responsible for Naphthenate Deposition in Crude Oil Processing. Org. Biomol. Chem. 2006, 4, 616-620.

17. Nishihara, M.; Morii, H.; Koga, Y. Structure Determination of a Quartet of Novel Tetraether Lipids from Methanobacterium thermoautotrophicum. J. Biochem. 1987, 101, 1007-1015.

18. Koga, Y; Morii, H. Special Methods for the Analysis of Ether Lipid Structure and Metabolism in Archaea. Anal. Biochem. 2006, 348, 1-14.

19. Schouten, S.; Huguet, C.; Hopmans, E. C.; Kienhuis, M. V. M.; Sinninghe

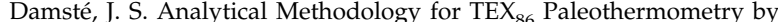
High-Performance Liquid Chromatography/Atmospheric Pressure Chemical Ionization-Mass Spectrometry. Anal. Chem. 2007, 79, 2940 2944.

20. Powers, L. A.; Werne, J. P.; Johnson, T. C.; Hopmans, E. C.; Damsté, J. S. S.; Schouten, S. Crenarchaeotal Membrane Lipids in Lake Sediments: A New Paleotemperature Proxy for Continental Paleoclimate Reconstruction? Geology 2004, 32, 613-616.

21. Pearson, A.; Huang, Z.; Ingalls, A. E.; Romanek, C. S.; Wiegel, J. Freeman, K. H.; Smittenberg, R. H.; Zhang, C. L. Nonmarine Crenarchaeol in Nevada Hot Springs. App. Environ. Microbiol. 2004, 70(9), $5229-5237$

22. Escala, M.; Rosell-Melé, A.; Masque, P. Rapid Screening of Glycerol Dialkyl Glycerol Tetraethers in Continental Eurasia Samples Using HPLC/APCI-Ion Trap Mass Spectrometry. Org. Geochem. 2007, 38 , 161-164.

23. Wasserfallen, A.; Nölling, J.; Pfister, P.; Reeve, J.; Conway de Macario, E. Phylogenetic Analysis of 18 Thermophilic Methanobacterium Isolates Supports the Proposals to Create a New Genus, Methanobacter gen. nov. and to Reclassify Several Isolates in Three Species, Methanothermobacter thermautotrophicus comb. nov., Methanobacter wolfeii comb. nov., and. Methanobacter marburgensis sp. nov. Int. J. Syst. Evol. Microbiol. 2000, 50, 43-53.

24. Galliker, P.; Gräther, O.; Rümmler, M.; Fitz, W.; Arigoni, D. New Structural and Biosynthetic Aspects of the Unusual Core Lipids from Archaebacteria. In Vitamin B12 and B12-proteins, Krautler, B., Arigoni, D., Golding, B. T., Eds.; Wiley-VCH: Hoboken, 1998, p. 447-458.

25. Wilson, M. A.; Ph.D. Thesis, University of York, UK, 2004. 